\title{
Probabilistic thermal-mechanical fatigue criterion for lost foam casting aluminium alloys based on 2D/3D porosities distribution
}

\author{
F. Szmytka ${ }^{1, a}$, N. Limodin ${ }^{2}$, L. Wang ${ }^{2}$, P. Osmond ${ }^{3}$, J. Adrien ${ }^{4}$, E. Charkaluk ${ }^{2}$ and J.Y. Buffiere ${ }^{4}$ \\ ${ }^{1}$ PSA Peugeot Citroën, Route de Gisy, 78643 Vélizy-Villacoublay, France \\ ${ }^{2}$ LML, Ecole Centrale de Lille Cité Scientifique 59650 Villeneuve d'Ascq, France \\ ${ }^{3}$ PSA Peugeot Citroën, Rue des Fauvelles, 92256 La Garenne Colombes, France \\ ${ }^{4}$ MATEIS, INSA Lyon, 69621 Villeurbanne Cedex, France
}

\begin{abstract}
A thermal-mechanical fatigue criterion is proposed to assess the lifetime of aluminium alloys produced by a lost foam casting process. It is based on the observed size distribution of pores sizes which are considered as critical zones for cracks initiation and gives reliable results when both pores sizes are identified from X-ray tomography and mean stresses are taken into account. Lifetime probabilities are finally successfully compared with experimental results.
\end{abstract}

Nowadays, car engines tend to be exposed to very high temperature and pressure during their nominal use, to improve their performance and to reduce their environmental impact. This trend requires the use of new materials or new processes for avoiding thermal-mechanical fatigue (TMF) damage in cylinder heads that are subjected to increasing temperatures and stresses. Lost Foam Casting (LFC) is a process that enables to easily adapt the geometry of cast structures against TMF with a reduced cost. It is now widely used in the automotive industry to produce cylinder heads out of aluminium alloys. However, its main drawback lies in the presence of more numerous pores and intermetallics, defects that can help to initiate TMF cracks. Pores are here taken as the defect population on which TMF cracks initiate.

The studied materials are two aluminum-silicon alloys used in the automotive industry: A356 with T7 heat treatment and A319 without heat treatment. This study focuses essentially on the materials obtained by LFC process which has a great impact on the microstructure of the materials as it was already illustrated in [1]. Fatigue data have been obtained on over-aged materials to ensure stable mechanical behaviour. The over-aging has been determined by the stabilisation both in terms of mechanical properties and of microstructure of the material which corresponds here to the heating of materials at $250^{\circ} \mathrm{C}$ for $200 \mathrm{~h}$. A full 3D characterization of the microstructure through X-ray tomography [2] is performed in order to measure the size and shape distribution of pores in a Representative Elementary Volume while 2D analyses are also carried out on samples cut from the same cylinder

\footnotetext{
${ }^{a}$ Corresponding author: fabien.szmytka@mpsa.com
}

This is an Open Access article distributed under the terms of the Creative Commons Attribution License 4.0, which permits unrestricted use, distribution, and reproduction in any medium, provided the original work is properly cited. 


\section{MATEC Web of Conferences}

heads to assess the validity of 2D metallographic analysis compared to the $3 \mathrm{D}$ characterization provided by Computed Tomography. A quantitative description of the pore population is obtained through the measurement of their Feret diameter and sphericity, which reveal the presence of gassing porosities which are not observed in standard die casting processes. The largest and sharpest pores have to be accurately observed as they may play an important part in the fatigue crack initiation. Study of pore size histograms in 2D and 3D shows indeed the inability of 2D analysis to detect of large pores (above $500 \mu \mathrm{m}$ ) although smaller pores are statistically well described. 2D analyses also tend to sometime overestimate the presence of very small pores that usually lead to bad description of the probability of failure for large number of cycles. The porosities distributions are then introduced in the computational approach used to design engine parts against TMF [3]. Under a basic assumption of growth equation of pores from an initial size $a_{0}$ to a final size $a_{f}$ at $N_{f}$ characterizing a macroscopic crack initiation, we develop a fatigue criterion under a probabilistic form as proposed by [4]. Here, a different damage indicator, as proposed by [5], enables to take into account different kinds of micro-cracks propagation and leads to the following improved form of the fatigue criterion:

$$
N_{f}=\int_{a_{o}}^{a_{f}} \frac{1}{\left[\left(\frac{w^{d}}{k_{1}}\right)^{n_{1}} a^{n_{1}}+\left(\frac{w^{e}}{k_{2}}\right)^{n_{2}} a^{n_{2}}\right]} d a
$$

where $w^{d}$ and $w^{e}$ denotes respectively inelastic and elastic dissipated energy densities per stabilized cycle, and $k_{1}, n_{1}, k_{2}$ and $n_{2}$ are material constants. $a$ consists in the observed statistical distribution of pore size while material parameters are identified from the macroscopic Low-Cycle Fatigue and TMF experiments. $N_{f}$ and $a$ are thus random variables. The numerical identification of the parameters of such a model is quite complex because no analytical description is possible. Treatment is then carried out by class of pore size. For a given pore size and for a given set of parameter $\left(k_{1} ; n_{1} ; k_{2} ; n_{2}\right)$, the estimated lifetime is easily calculated. A histogram and a complete distribution of the number of cycles to failure are then obtained. For a given experimental couple $\left(w^{d} ; w^{e}\right)$, the number of cycles to failure with the highest probability is then finally compared to the experimental lifetime. A leastsquares optimization is then performed to obtain the most relevant parameter set. Lifetime prediction results are compared on both laboratory and technological (simplified structure) specimens for 2D and 3D analysis of pores. Lifetime distributions obtained by the present method and by [3] and [4] are compared. The direct prediction results show a small improvement when compared with the usage of a standard fatigue criterion in [3] but the main benefit of the proposed criterion is, as in [4], the estimation of a probability density function both as a simple relation between microstructure distribution and macroscopic lifetime observations and as a complement to a statistical distribution of tests which is generally very expensive. The proposed criterion tends to show more realistic lifetime distribution than in [3] and provide satisfying results for A319 and A356 LFC aluminium alloys.

\section{References}

[1] S. Tabibian, E. Charkaluk, A. Constantinescu, A. Oudin, F. Szmytka, Procedia Engineering 2, Issue 1, April 2010, Pages 1145-1154

[2] N. Limodin, A. El Bartali, L. Wang, J. Lachambre, J.-Y. Buffiere, E. Charkaluk, Nuclear Instruments and Methods in Physics Research Section B: Beam Interactions with Materials and Atoms 324, April 2014, pages 57-62

[3] A. Constantinescu A., E. Charkaluk, G. Lederer, L. Verger, International Journal of Fatigue 26, 2004, 8, pages 805-818.

[4] E. Charkaluk, A. Constantinescu, F. Szmytka, S. Tabibian, International Journal of Fatigue 63, 2014, pages 127-136.

[5] V. Maurel, L. Rémy, F. Dahmen, N. Haddar, International Journal of Fatigue 31, Issue 5, May 2009, Pages 952-961 